\title{
EMERGÊNCIA DE CLASSES ORDINAIS APÓS O ENSINO DE RELAÇÕES NUMÉRICAS ${ }^{I}$
}

\section{THE EMERGENCE OF ORDINAL CLASSES DERIVED FROM TEACHING NUMERIC RELATIONS}

\author{
Ana Letícia de Moraes Nunes e Grauben José Alves de Assis \\ UNIVERSIDADE FEDERAL DO PARA, BRASIL
}

\begin{abstract}
RESUMO
O paradigma de equivalência tem se mostrado útil na explicação de processos comportamentais complexos, como aqueles envolvidos em comportamentos conceituais numéricos. O objetivo do presente trabalho foi verificar se classes ordinais poderiam emergir após o ensino por emparelhamento arbitrário e de produção de seqüência. Três alunos com atraso no desenvolvimento foram expostos a estímulos visuais de formas abstratas indicando numerosidade (A), numerais (B) e nomes escritos de numerais (C). Depois que as relaçôes AB/AC foram ensinadas testou-se a emergência de três classes de equivalência. Em seguida foi ensinado o encadeamento de respostas com estímulos de um dos conjuntos (A1 $\rightarrow \mathrm{A} 2 \rightarrow \mathrm{A} 3)$ e avaliada a emergência de novas seqüências $(\mathrm{B} 1 \rightarrow \mathrm{B} 2 \rightarrow \mathrm{B} 3$ e $\mathrm{C} 1 \rightarrow \mathrm{C} 2 \rightarrow \mathrm{C} 3)$. Posteriormente, foram conduzidos testes de conectividade para verificar a formaçáo de classes ordinais (por exemplo: $\mathrm{A} 1 \rightarrow \mathrm{B} 2 \rightarrow \mathrm{C} 3$ ). Testes de generalização também eram apresentados para verificar se um responder envolvendo numerosidade ocorreria com novos estímulos (por exemplo: E1 $\rightarrow \mathrm{E} 2 \rightarrow \mathrm{E} 3$ ). Os resultados demonstraram que os participantes responderam a novas seqüências prontamente ou com emergência gradual, inclusive nos testes de generalização. O procedimento mostrou-se também eficiente na transferência de funções ordinais em pessoas com atraso no desenvolvimento.
\end{abstract}

Palavras-chave: equivalência, seqüências, classes ordinais, relaçôes numéricas, atraso no desenvolvimento, educação matemática.

\section{ABSTRACT}

The equivalence paradigm has been useful in the explanation of complex behavioral processes such as those involved in numeric conceptual behaviors. The purpose of the present study was to verify whether ordinal classes could emerge following arbitrary matching-to-sample and sequence production. Participants were three students with developmental disabilities. The visual stimuli were abstract forms in different quantities (A), cardinal numbers $(B)$ and written words in capital letters $(\mathrm{C})$. The $\mathrm{AB} / \mathrm{AC}$ relations were trained and the emergence of three equivalence classes was tested. After the forward chaining procedure to teach one sequence $(A 1 \rightarrow A 2 \rightarrow A 3)$, the emergence of novel sequences was assessed (e. g. B1 $\rightarrow$ B2 $\rightarrow$ B3 and $\mathrm{C} 1 \rightarrow \mathrm{C} 2 \rightarrow \mathrm{C} 3$ ). Connectivity tests assessed the ordinal classes formation (e.g. $\mathrm{A} 1 \rightarrow \mathrm{B} 2 \rightarrow \mathrm{C} 3$ ). Results showed that the participants responded to novel sequences readily or with gradual emergence. All participants responded to sequences with new stimuli. The procedure was also effective in the transfer of ordinal functions in persons with developmental disabilities.

Keywords: equivalence, sequences, ordinal classes, numeric relations, developmental disabilities, math education.

O procedimento de emparelhamento de acordo com o modelo (matching-to-sample MTS), consiste em um arranjo do procedimento de discriminação condicional. Nesse procedimento um estímulo modelo é apresentado ao participante e após emissão de uma resposta ao modelo, são apresentados dois ou mais estímulos de comparação. A resposta ao estímulo de comparação correto é seguida por reforço. De acordo com Cumming e Berryman (1965), enquanto na discriminação simples o estímulo discriminativo controla respostas específicas, na discriminação condicional a função do estímulo discriminativo muda de acordo com o contexto. Assim, no procedimento de emparelhamento de acordo

1 Agradecemos as sugestões valiosas dos professores Dr. Paulo Sérgio Teixeira Prado (Unesp-Marília) e Dr. Olavo de Faria Galvão (UFPA) e ao programador João Vicente Cunha Nascimento pelo apoio técnico na confecção do software. Endereço para correspondência:Trav. Castelo Branco, $1923 / 301$ Bairro do Guamá, Belém-Pará. CEP: 66.063.420 - Fone/Fax: 91-3201-7662. E-mail: grauben@pesquisador.cnpq.br 
com o modelo, o reforço é contingente à relação entre os estímulos modelo (condicionais) e de comparação (discriminativos). O estímulo modelo funcionaria como um estímulo "instrucional”, que selecionaria o estímulo de comparação com função de estímulo discriminativo num dado momento.

Sidman (1986) descreveu a contingência de quatro termos como a unidade fundamental do que pode-se chamar de controle de estímulo condicional. Uma relevância particular dessa unidade de análise é a de favorecer a emergência de relações de equivalência. Por meio de um procedimento padrão de emparelhamento de acordo com o modelo, podem-se estabelecer relações condicionais e depois testá-las. Dessa forma, podem emergir novas unidades comportamentais nas quais os estímulos relacionados uns aos outros se tornam membros de uma classe, mesmo que para o organismo não tenham sido ensinadas discriminações condicionais adicionais.

O fenômeno de equivalência constitui um processo comportamental básico (Sidman, 1994) que requer propriedades relacionais (reflexividade, simetria, transitividade e equivalência), baseadas na definição matemática de equivalência (Sidman $\&$ Tailby, 1982). A reflexividade pode ser inferida em uma tarefa de matching generalizado, em que há uma relação de identidade entre os estímulos. A simetria indica que uma relação entre estímulos é bidirecional. O estímulo modelo e o estímulo de comparação devem apresentar funções intercambiáveis, ou seja, o estímulo modelo poderá funcionar como estímulo de comparação e vice-versa no contexto de um emparelhamento arbitrário de acordo com o modelo (matchingto-sample-MTS), portanto, uma reversibilidade funcional. O terceiro requisito para equivalência é que as relações condicionais sejam transitivas. Por exemplo, dado que o indivíduo foi capaz de relacionar $\mathrm{AB}$ e $\mathrm{BC}$, ele deveria conseqüentemente estabelecer uma relação $\mathrm{AC}$, na qual $B$ funciona como nódulo, ou seja, um elo de ligação entre as relações $\mathrm{AB}$ e $\mathrm{BC}$.

Portanto, AC é uma relação de transitividade. A emergência de uma outra relação (CA) indica que a relação é de equivalência por implicar na simetria e na transitividade. A partir do ensino direto de apenas duas relações, quatro novas relações poderão emergir sem qualquer ensino adicional. Isso implica, para a área educacional, uma maior eficiência e economia durante o processo de ensino.

Uma análise baseada no paradigma de equivalência permite compreender como estímulos fisicamente diferentes podem se tornar substituíveis no controle de determinadas respostas (cf. Sidman, 1994). Observa-se adiante como a substituição de estímulos, em que um membro de uma classe exerce a mesma função dos demais membros da mesma classe, pode ser verificada em um outro contexto.

Green, Stromer e Mackay (1993) propuseram um novo tipo de análise de desempenhos emergentes derivados de contingências que estabelecem a produção de sequiência. Tais autores fazem uma extensão da análise de relações entre estímulos em classes de equivalência proposta por Sidman e Tailby (1982). O enfoque está nas relações entre estímulos em seqüência e entre seqüências ensinadas separadamente. Testes comportamentais avaliam se tais relações apresentam as propriedades de uma relação ordinal: irreflexividade, assimetria, transitividade e conectividade (Stevens, 1951).

A irreflexividade é uma relação ordinal explicitamente não reflexiva, em que 
$\mathrm{A} 1 \rightarrow \mathrm{A} 1$ (A1 é seguido por A1) é falsa. A propriedade da assimetria pressupõe uma relação de ordem unidirecional (se $\mathrm{A} 2 \rightarrow \mathrm{A} 3$, então A3 $\rightarrow$ A2 é falsa). A transitividade pode ser verificada por meio de pares de estímulos não adjacentes, por exemplo, se $\mathrm{A} 2 \rightarrow \mathrm{A} 3 \mathrm{e}$ $\mathrm{A} 3 \rightarrow \mathrm{A} 4$, então A2 $\rightarrow$ A4. A conectividade é inferida se todos os pares possíveis dentro de uma série estão em uma ordem consistente.

Segundo Green et al. (1993), os conceitos de cadeias simples e controle condicional não são suficientes para explicar desempenhos emergentes dentro de uma seqüência e entre seqüências ensinadas separadamente. Assim, a questão pertinente é: como um organismo pode responder ordinalmente a eventos fisicamente diferentes que se sucedem no tempo e não foram previamente relacionados? A proposta de Green et al. (1993) permite investigar as possíveis fontes de controle de comportamentos emergentes, diferente de um contexto que envolva a emergência de relaçôes condicionais (emparelhamento de acordo com o modelo), como proposto por Sidman e Tailby (1982).

Os procedimentos de produção de seqüência consistem na apresentação simultânea de dois ou mais estímulos visuais arbitrários e contingências de reforçamento que requerem respostas de apontar aos estímulos em uma dada ordem, independente da posição espacial (c.f. Green, Sigurdardottir, \& Saunders, 1991; Lazar, 1977; Sigurdadottir, Green, \& Saunders, 1990; Stromer \& Mackay, 1993). As duas formas propostas para o estabelecimento de seqüências comportamentais são: encadeamento (chaining) e pares de estímulos sobrepostos (overlapping two-stimuli sequences). No encadeamento os estímulos são adicionados gradativamente até completar uma dada seqüência (ex: $A 1 \rightarrow A 2, A 1 \rightarrow A 2 \rightarrow A 3$, etc.). Já o ensino por meio de pares justapostos é realizado com a apresentação simultânea de estímulos adjacentes de uma dada seqüência (ex: A1 $\rightarrow$ A2, A2 $\rightarrow$ A3; etc.).

Uma das táticas empregadas para examinar as propriedades ordinais e a formação de classes ordinais é o ensino de pelo menos duas seqüências independentes e testes entre seqüências. Por exemplo, após o ensino da seqüência "A" (A1 $\rightarrow$ A2 $\rightarrow$ A3 $\rightarrow$ A $4 \rightarrow \mathrm{A} 5)$ e da seqüência "B" $(\mathrm{B} 1 \rightarrow \mathrm{B} 2 \rightarrow \mathrm{B} 3 \rightarrow \mathrm{B} 4 \rightarrow \mathrm{B} 5)$, Stromer e Mackay (1993) aplicaram testes de conectividade (e.g. A1 $\rightarrow$ B2 $\rightarrow$ A3 $\rightarrow$ B $4 \rightarrow$ A5) para a verificação da formação de classes ordinais.

Outra tática usada para investigar a possibilidade de formação de classes ordinais e examinar as propriedades das relações de ordem é o ensino de uma seqüência com membros de classes de equivalência estabelecidas. Após o estabelecimento de classes de estímulos equivalentes, os participantes são ensinados a ordenar estímulos consistindo de um membro de cada classe de equivalência. Os testes, então, avaliam se os participantes respondem funcionalmente a relações de ordem, produzindo novas seqüências, sem qualquer ensino adicional. Por exemplo, após o ensino de relações condicionais $\mathrm{AB}$ e $\mathrm{AC}$ e da produção da seqüência "A", pode-se testar a emergência de desempenhos sob controle das seqüências "B" e "C". A produção de novas sequiências pela substituição de estímulos equivalentes deve implicar que o ensino da seqüência " $A$ " estabeleceu relações de ordem e que os demais membros das classes de equivalência também passam a exercer funçôes ordinais ("primeiros", "segundos", etc.). Portanto, a substitutabilidade de estímulos em seqüências pode ser examinada com essa tática, segundo Green et al. (1993).

Dois estudos experimentais (Kotlarchyk, 
\& Stromer, 1997; Maydak, Stromer, Mackay, \& Stoddard, 1995; Mackay,) envolveram uma análise pormenorizada da emergência de relações condicionais e relações ordinais com estímulos numéricos. Uma particularidade a ser considerada é que em ambos os estudos os participantes apresentavam atraso no desenvolvimento cognitivo. Outra característica relevante nesses dois estudos é que relações condicionais já estabelecidas envolvendo estímulos familiares eram algumas das relações pré-requisitos para a emergência de novos comportamentos. Tem sido sugerido que a adição de estímulos em classes já existentes pode ocorrer de forma mais rápida do que na formação de classes, a partir das tarefas experimentais (O’Donnel \& Saunders, 2003). Por esse motivo, no presente estudo as relações arbitrárias ensinadas não faziam parte do repertório dos participantes.

Maydak et al. (1995) investigaram as interrelações entre classes de estímulos estabelecidas pelo emparelhamento de acordo com o modelo e tarefas de produção de seqüência realizadas por dois indivíduos com atraso no desenvolvimento. A questão principal era se classes de estímulos inicialmente demonstradas no contexto de emparelhamento de acordo com o modelo forneceriam a base para a emergência de desempenhos não ensinados e em outros contextos. Os estímulos usados foram palavras ditadas de numerais cardinais, numerais impressos, conjuntos de pontos (quantidade) e formas abstratas. O procedimento consistiu no ensino e teste de relaçôes condicionais por emparelhamento por identidade e arbitrário com estímulos de quantidades e numerais; e emparelhamento arbitrário de numerais e formas abstratas. A outra tarefa, ensinada pelo procedimento de fading, consistiu na produção de uma seqüência com conjuntos de pontos em diferentes quantidades na ordem crescente. Houve variabilidade nos resultados. Um dos participantes atingiu os critérios na linha de base e desempenhos considerados satisfatórios nos testes de equivalência e de produção de seqüência de numerais e formas abstratas. $\mathrm{O}$ segundo participante alcançou com dificuldade a linha de base de emparelhamento de acordo com o modelo e apresentou um baixo índice de acerto em testes de produção de seqüência. Uma das questôes identificadas é que nos testes de equivalência havia reforçamento diferencial e isso pode ter afetado o desempenho de um participante, já que o mesmo apresentava alguns dos pré-requisitos dos testes antes do experimento. Os autores ressaltaram que a definição dos pré-requisitos necessários nas tarefas de produção de seqüência para transferência de classes em emparelhamento arbitrário necessitava de estudos adicionais. Além disso, não foram verificadas as propriedades de relações ordinais entre seqüências não ensinadas (cf. Green et al., 1993).

Mackay et al. (1997) ensinaram uma criança com paralisia cerebral a ordenar letras formando palavras, por meio do ensino por resposta construída (anagram construction), em que o estímulo modelo nessa tarefa era uma palavra ditada de um número e a tarefa consistia em selecionar as letras apresentadas no painel de escolha. Esse estudo permitiu a ampliação do repertório de emparelhamento de acordo com o modelo, baseado em uma rede de relações e de nomeação de numerais, palavras escritas e seqüência oral de "zero" a "nove". Um ensino de seqüenciação de numerais impressos (de $0 \mathrm{a}$ 3) foi realizado no segundo estudo, ampliando as classes. Esses resultados corroboram a 
hipótese de que o ensino de uma seqüência com um estímulo de cada classe de equivalência pode levar os demais estímulos de cada classe a adquirirem a mesma função ordinal.

A partir dos resultados dos estudos aqui descritos, pesquisas adicionais seriam necessárias para identificar ou explicitar fontes de controle sobre as relações entre classes de equivalência e classes ordinais. Quais as propriedades e funções de eventos ambientais no controle desses comportamentos?

A substitutabilidade de estímulos que ocorre no formato de emparelhamento de acordo com o modelo também pode ser demonstrada no contexto de produção de seqüências em contingências de três termos? Resultados anteriores com pessoas com atraso no desenvolvimento poderiam ser replicados?

As pesquisas têm buscado compreender como desempenhos sob controle da função de ordem são estabelecidos, como é mantido seu potencial produtivo e quais as condições relevantes para a formação de classes ordinais. Verifica-se também se os estímulos são funcionalmente equivalentes nos testes de conectividade entre seqüências.

Esses estudos são úteis para explicar comportamentos complexos como o comportamento conceitual numérico. $\mathrm{O}$ foco principal do estudo é a análise da relação entre desempenhos de emparelhamento arbitrário e de produção de seqüências numéricas.

O objetivo do presente trabalho foi investigar se classes ordinais poderiam emergir após o ensino por emparelhamento arbitrário de acordo com o modelo e produção de seqüências. Ou seja, classes de estímulos equivalentes poderiam fornecer a base para a emergência de classes ordinais? Classes ordinais também poderiam se transferir para novos contextos? A transferência é observada quando uma variável aplicada a um dado membro da classe afeta, do mesmo modo, os demais membros da classe.

\section{MÉTODO}

\section{Participantes}

Participaram do estudo, três alunos com atraso no desenvolvimento em fase de alfabetização, dois portadores de Síndrome de Down do sexo masculino, de 14 e 24 anos de idade e um com atraso no desenvolvimento neuropsicomotor, do sexo feminino, de 17 anos. A seleção dos participantes foi realizada com base nos desempenhos apresentados no préteste. Os responsáveis pelos participantes eram informados que se tratava de uma pesquisa sobre métodos de ensino de conceitos matemáticos. Um termo de consentimento livre e esclarecido era assinado pelo responsável, autorizando a participação do aluno no estudo conforme exigência da Resolução 196/96 do Conselho Nacional de Saúde.

\section{Ambiente experimental e materiais}

Os materiais utilizados nos pré-testes foram um jogo de peças madeira com numerais cardinais, conjuntos de elementos e nomes escritos dos numerais, o Instrumento de Avaliação do Repertório Básico para Alfabetização (Leite, 1984), e o Peabody Picture Vocabulary Test (Dunn \& Dunn, 1981).

As sessões foram realizadas em uma sala de pesquisa de uma escola especial (APAE Belém). O participante sentava-se em frente a um computador e o experimentador ficava ao seu lado, monitorando a sessão experimental. Nas sessōes experimentais, o equipamento era um microcomputador 
Pentium de $300 \mathrm{MHz}$, usado para controle da apresentação dos estímulos, números de tentativas, números de posições de cada estímulo na tela e registro das respostas corretas e incorretas. O programa utilizado (REL - relações entre estímulos, na versão 3.0) foi elaborado em linguagem VISUAL BASIC 6.0 por João Vicente do Nascimento.

O conjunto designado pela letra "A", era constituído de formas abstratas em diferentes quantidades. $\mathrm{O}$ conjunto " $\mathrm{B}$ " era de numerais cardinais. O conjunto " $\mathrm{C}$ " era de palavras escritas de numerais cardinais. Outras formas abstratas de quantidades também eram utilizadas na fase de generalização.

\section{Procedimento geral}

Pré-testes foram aplicados inicialmente para se verificar o repertório numérico dos participantes. Foi aplicado o Peabody Picture Vocabulary Test (Dunn \& Dunn, 1981), para avaliar o nível de desempenho equivalente, a partir do repertório verbal do participante. O Instrumento de Avaliação de Repertório Básico para Alfabetização (IAR) também foi aplicado para verificar repertórios relativos à lateralidade, discriminação auditiva, visual, orientação espacial, noção de conceitos relacionais abstratos, como: acima, abaixo, etc. (Leite, 1984).

O procedimento foi dividido em quatro fases gerais: emparelhamento por identidade, emparelhamento arbitrário, seqüências e generalização. Nos pré-testes, nas sondas e nos testes, as respostas não eram conseqüenciadas. Durante as sessões de ensino, as respostas corretas eram conseqüenciadas com reforço verbal fornecido pelo pesquisador, com a apresentação de uma animação gráfica e de um som gerado pelo computador (aplausos).
Emparelhamento de acordo com o modelo. Todas as fases do ensino de emparelhamento de acordo com o modelo foram dispostas em blocos de tentativas. A instrução era: "Toque na figura”. A resposta do participante era a de tocar na tela, em cima do estímulo modelo que era apresentado no interior de uma "janela" de $5,7 \mathrm{~cm}$ por $5,7 \mathrm{~cm}$. Tocar no modelo produzia um, dois ou três estímulos de comparação, dependendo da fase de ensino, dispostos no interior de cada uma das 4 janelas laterais (uma "janela" permanecia desativada). Os estímulos de comparação eram introduzidos gradativamente, até atingirem três estímulos simultaneamente.
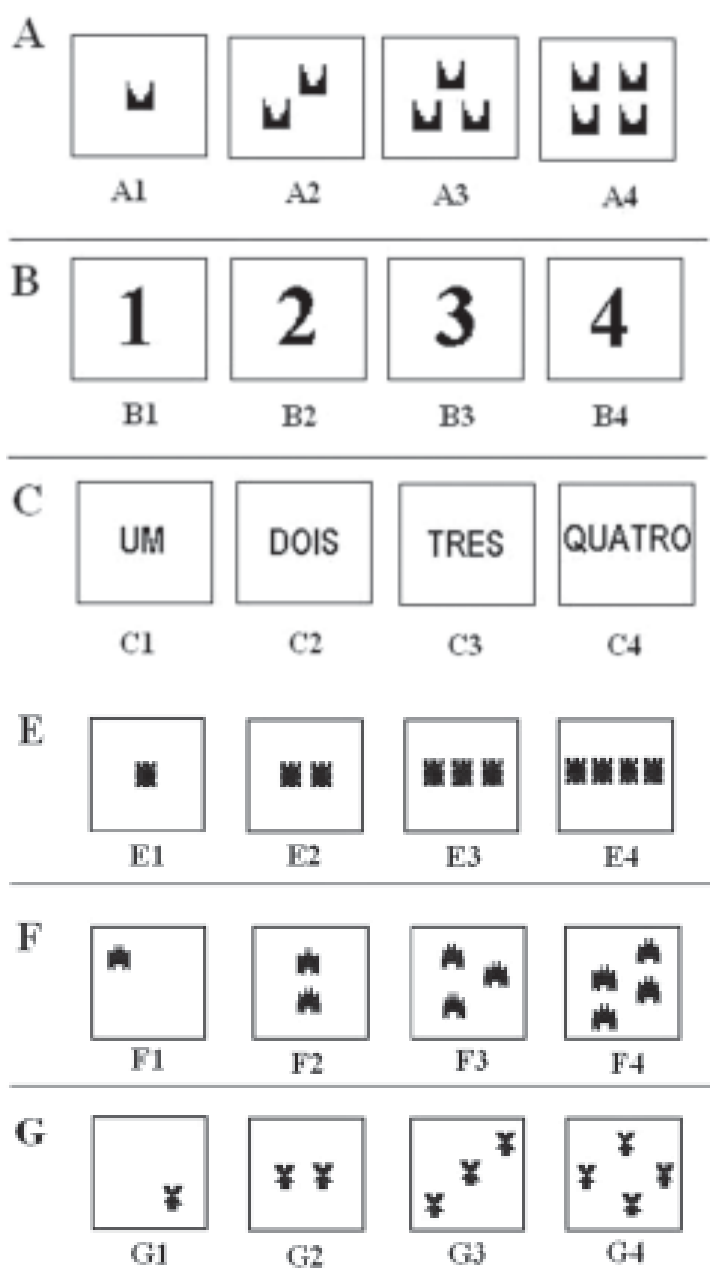

Figura 1: Conjuntos de estímulos empregados no estudo. 
Uma relação era ensinada com bloco de 12 tentativas, ou seja, um mesmo modelo era apresentado em todas as tentativas. Em seguida, um outro modelo era apresentado com mais 12 tentativas. Atingindo-se o critério de acerto de $100 \%$ por bloco, havia a redução do número de tentativas para seis e depois três com o mesmo modelo até a apresentação de um bloco misto com três modelos diferentes apresentados alternadamente. Os blocos de linha de base eram com todas as relações já ensinadas com três estímulos de comparação, apresentados de forma randômica (cf. Saunders \& Spradlin, 1990).

O participante deveria tocar em um estímulo de comparação considerado correto $\left(S_{+}\right)$. A probabilidade de reforço nos blocos de ensino era de 1,0. O pesquisador controlava o mouse e clicava onde o participante tocasse na tela. Caso o participante respondesse corretamente, uma animação gráfica era apresentada na tela do computador com o som de aplausos, com duração de 3 s e o pesquisador conseqüenciava com um reforço verbal (e. g. "Muito bem”, "legal”, “certo”).

Em seguida, uma nova tentativa era iniciada. Caso a resposta fosse incorreta, não havia conseqüência reforçadora e a mesma configuração de estímulos era apresentada na tela (procedimento de correção). Se o participante tocasse em alguma janela desativada, não havia conseqüência reforçadora e a configuração de estímulos permanecia até que ocorresse a resposta de tocar em um estímulo de comparação. A posição dos estímulos de comparação mudava de tentativa para tentativa, para evitar o efeito do controle pela posição dos estímulos de comparação. Na programação dos blocos, foi feito um balanceamento das tentativas apresentadas. Cada bloco tinha 12 tentativas. O bloco de sonda tinha seis tentativas e o objetivo era verificar se o responder permanecia estável mesmo na ausência do reforçamento diferencial.

Os blocos de linha de base eram com todas as três relações já ensinadas alternadamente com três estímulos de comparação. O critério de acerto era de 100\% por bloco. Tendo alcançado esse critério, ocorria a exposição ao bloco seguinte. Se o participante não atingisse o critério, era reexposto ao mesmo bloco até 4 vezes, no máximo. Todos os blocos de ensino de emparelhamento de acordo com o modelo (identidade e arbitrário) tinham o mesmo critério de acerto.

Inicialmente, o ensino era de emparelhamento por identidade, com testes de identidade generalizada. O objetivo era instalar a discriminação condicional por identidade. Esse procedimento foi introduzido devido às evidências de que o desempenho de emparelhamento por identidade constitui um pré-requisito para desempenhos de emparelhamento arbitrário (c.f. Dube, 1996). $\mathrm{Na}$ segunda fase, aplicou-se um procedimento de ensino de emparelhamento arbitrário, com a finalidade de se estabelecer o desempenho de discriminação condicional AB. Após o alcance do critério de linha de base, era aplicado o teste de simetria BA. Depois, aplicou-se o ensino de emparelhamento arbitrário $\mathrm{AC}$, seguido dos testes de simetria CA. Após a retomada da linha de base $\mathrm{AB} / \mathrm{AC}$, foram aplicados os testes de transitividade e equivalência BC e CB.

\section{Encadeamento e testes de produção de} seqüências. Nesta fase era realizado um ensino por encadeamento para frente com o conjunto de estímulos "A". Nesse tipo de ensino, os estímulos eram adicionados um a um. O 
participante deveria responder ordinalmente. A matriz utilizada era dividida em duas áreas: área de "escolha" e a área de "construção". $\mathrm{Na}$ área de escolha eram apresentados os estímulos que o participante deveria selecionar um após o outro. Após selecionar um estímulo, este se deslocava para a área de construção onde permanecia em uma das "janelas" da esquerda para a direita. Cada tentativa era iniciada com a apresentação de uma "janela" cinza escrita "Toque", na parte superior da tela. Ao tocar nessa "janela", o primeiro estímulo (A1) aparecia em uma das oito "janelas" da área de escolha, na parte inferior da tela. O participante deveria tocar nesse estímulo. $\mathrm{O}$ pesquisador fornecia as seguintes instruções ao participante: "Vai aparecer uma figura" (o pesquisador apontava a parte inferior da tela). "Toque na figura" (o pesquisador esperava o participante tocar na figura). Um toque na figura produzia seu deslocamento da "área de escolha" para a primeira das oito "janelas" (da esquerda para a direita) da "área de construção" (acima da área de escolha). Após a resposta correta do participante, uma animação gráfica aparecia na tela do computador, juntamente com o som de aplausos e o reforço verbal fornecido pelo pesquisador (e.g. "Muito bem!", "legal”, "Parabéns!"). Em seguida, um segundo estímulo era adicionado em qualquer uma das "janelas" da área de escolha. O participante deveria tocar primeiro A1 e depois A2. A instrução era a seguinte: "Vão aparecer as figuras na tela. Toque em uma figura e depois na outra". O critério de acerto era a formação correta da sequiência três vezes consecutivas, sem erro. Quando a resposta era incorreta (por exemplo, $A 2 \rightarrow A 1$ ), não havia conseqüência reforçadora, a tentativa se encerrava e os mesmos estímulos reapareciam na "área de escolha" na mesma posição (procedimento de correção), após um intervalo de 3s. O participante era exposto à mesma seqüência até 10 vezes, no máximo. Após alcançar o critério de acerto, uma outra figura era adicionada na área de escolha e assim sucessivamente até completar quatro figuras. $\mathrm{O}$ estímulo A4 funcionava como um estímulo "distrator", para que a resposta ao terceiro estímulo da seqüência (A3) não fosse por exclusão e era apresentado da mesma forma que os demais. A matriz de escolhas pode ser visualizada na Figura 2.

Após cada estímulo ser adicionado à seqüência, havia uma sonda com uma tentativa de ensino, mas sem reforçamento diferencial. Após o ensino $\mathrm{A} 1 \rightarrow \mathrm{A} 2$, havia uma sonda $\mathrm{A} 1 \rightarrow \mathrm{A} 2$; após o ensino $\mathrm{A} 1 \rightarrow \mathrm{A} 2 \rightarrow \mathrm{A} 3$, havia a sonda $A 1 \rightarrow A 2 \rightarrow A 3$ e assim sucessivamente. Se o participante respondesse diferente do programado pela experimentadora, havia uma segunda oportunidade. Antes de apresentar a sonda, dizia-se ao participante: "Você deverá tocar uma figura de cada vez. Agora não haverá aplausos e nem o Mickey, mas o computador continuará gravando suas respostas. Você entendeu?”.

Em seguida, uma revisão da linha de base do ensino das relações condicionais $\mathrm{AB} / \mathrm{AC}$ era aplicada. $\mathrm{Na}$ fase seguinte, os participantes eram expostos ao teste de produção de sequiência com estímulos do conjunto "B" (B1 $\rightarrow \mathrm{B} 2 \rightarrow \mathrm{B} 3 / \mathrm{B} 4)$. No teste de produção de sequiência, o participante deveria responder ordinalmente sem reforçamento diferencial. Dada a história anterior de ensino por emparelhamento arbitrário, esse teste tinha como objetivo avaliar se estímulos equivalentes passariam a exercer funções ordinais (de acordo com o ensino da seqüência "A"), sem qualquer ensino adicional. Posteriormente, aplicava-se o teste de produção de sequiência com estímulos do conjunto "C" $(\mathrm{C} 1 \rightarrow \mathrm{C} 2 \rightarrow \mathrm{C} 3 / \mathrm{C} 4)$. 


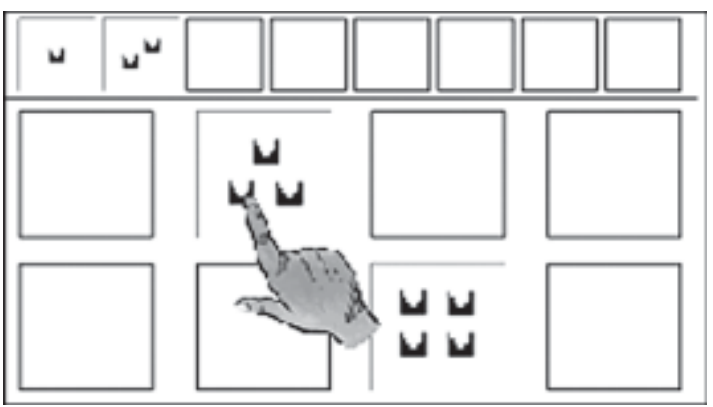

Figura 2: Matriz usada na fase de ensino e testes de produção de seqüência.

A seguir foram aplicados os testes de conectividade com estímulos dos conjuntos "A", "B" e "C". Durante esses testes, as seqüências "A", "B" e "C" eram misturadas, por exemplo, $\mathrm{A} 1 \rightarrow \mathrm{B} 2 \rightarrow \mathrm{C} 3 / \mathrm{C} 4$. Esses estímulos eram apresentados aleatoriamente em janelas dispostas lado a lado. Todos os estímulos estavam presentes na "área de escolha” e o pesquisador fornecia ao participante a mesma instrução dos blocos de sonda e de teste de seqüenciação. A tarefa do participante era a de tocar todos os estímulos apresentados até que nenhum mais restasse na "área de escolha”. O desempenho era considerado emergente se o participante tocasse todos os estímulos que formam a seqüência na ordem correta prevista. Os blocos previstos eram $\mathrm{A} 1 \rightarrow \mathrm{B} 2 \rightarrow \mathrm{C} 3 / \mathrm{C} 4$, $\mathrm{B} 1 \rightarrow \mathrm{C} 2 \rightarrow \mathrm{A} 3 / \mathrm{A} 4, \quad \mathrm{C} 1 \rightarrow \mathrm{A} 2 \rightarrow \mathrm{B} 3 / \mathrm{B} 4$, $\mathrm{B} 1 \rightarrow \mathrm{A} 2 \rightarrow \mathrm{C} 3 / \mathrm{C} 4, \mathrm{~A} 1 \rightarrow \mathrm{C} 2 \rightarrow \mathrm{B} 3 / \mathrm{B} 4$ e $\mathrm{C} 1 \rightarrow \mathrm{B} 2 \rightarrow \mathrm{A} 3 / \mathrm{A} 4$.

Esse teste tinha o objetivo de analisar a formação de classes ordinais derivadas dos ensinos por emparelhamento arbitrário e por encadeamento de respostas da seqüência "A". Dessa forma, poderia ser demonstrada a substitutabilidade funcional dos estímulos entre as seqüências "A", "B" e "C". Se as classes tivessem sido formadas os estímulos que exerciam a mesma posição na série ordinal deveriam tornar-se substituíveis uns pelos outros nas seqüências. Por último, a fase de generalização teve como objetivo verificar a emergência de um responder generalizado a numerosidades, utilizando-se novos estímulos (formas abstratas com diferentes numerosidades). Se houvesse um responder diferente do programado, os testes eram repetidos após a retomada da linha de base das relações condicionais $\mathrm{AB} / \mathrm{AC}$ e do ensino da seqüência com estímulos do conjunto " $A$ ".

A fase de teste de generalização teve como objetivo verificar a emergência de um responder generalizado a numerosidades, utilizando-se novos estímulos (formas abstratas em diferentes numerosidades) no contexto de produção de seqüência. Os testes previstos eram os seguintes: $\mathrm{E} 1 \rightarrow \mathrm{E} 2 \rightarrow \mathrm{E} 3 / \mathrm{E} 4, \quad \mathrm{~F} 1 \rightarrow \mathrm{F} 2 \rightarrow \mathrm{F} 3 / \mathrm{F} 4$, $\mathrm{G} 1 \rightarrow \mathrm{G} 2 \rightarrow \mathrm{G} 3 / \mathrm{G} 4, \mathrm{E} 1 \rightarrow \mathrm{F} 2 \rightarrow \mathrm{G} 3 / \mathrm{G} 4$, $\mathrm{E} 1 \rightarrow \mathrm{G} 2 \rightarrow \mathrm{F} 3 / \mathrm{F} 4, \quad \mathrm{~F} 1 \rightarrow \mathrm{G} 2 \rightarrow \mathrm{E} 3 / \mathrm{E} 4$, $\mathrm{F} 1 \rightarrow \mathrm{E} 2 \rightarrow \mathrm{G} 3 / \mathrm{G} 4, \quad \mathrm{G} 1 \rightarrow \mathrm{E} 2 \rightarrow \mathrm{F} 3 / \mathrm{F} 4$, $\mathrm{G} 1 \rightarrow \mathrm{F} 2 \rightarrow \mathrm{E} 3 / \mathrm{E} 4$. Estes testes eram aplicados após a revisão da seqüência $\mathrm{A} 1 \rightarrow \mathrm{A} 2 \rightarrow \mathrm{A} 3 / \mathrm{A} 4 \mathrm{e}$ da exposição à respectiva sonda.

\section{RESULTADOS}

O Peabody Picture Vocabulary Test (Dunn \& Dunn, 1981) foi empregado para avaliar a idade de desempenho verbal dos participantes DRA, LBA e IJA, que foi equivalente a 4 anos e 5 meses; 4 anos e 6 meses, e 6 anos e 10 meses, respectivamente. No IAR, as áreas avaliadas foram: lateralidade, posição, direção, espaço, tamanho, quantidade e discriminação visual. Dentre essas, as áreas de quantidade e discriminação visual foram aquelas em que os participantes apresentaram maior dificuldade. No pré-teste de habilidades numéricas, verificou-se que o participante DRA nomeou o numeral 1 e contou até duas unidades. LBA também contou até duas unidades. IJA também 
nomeou o numeral 1 e a contagem de um elemento. As respostas às demais figuras foram incorretas. Nenhum dos participantes apresentou comportamento de leitura com relação às palavras do pré-teste.

\section{EMparelHamento Arbitrário}

$\mathrm{Na}$ fase de ensino de emparelhamento arbitrário com os elementos dos conjuntos os participantes DRA e LBA foram ensinados a contar a quantidade de elementos dos estímulos modelo (conjunto "A"). IJA teve esse desempenho ensinado ainda nos blocos de discriminação condicional por identidade. $\mathrm{O}$ participante DRA respondeu prontamente na primeira exposição do bloco misto de linha de base (AB). No teste de simetria "BA" o participante atingiu o critério de acerto na segunda exposição ao teste. No ensino por emparelhamento arbitrário com os estímulos dos conjuntos "A" e "C" o participante acertou $11 / 12$ no terceiro e no quarto bloco. Ainda que não fosse $100 \%$ de acerto, esse desempenho foi considerado suficiente. Já que atingiu o critério de acerto da sonda, o participante passou para

\section{Tabela 1}

Número de acertos sobre o número de tentativas nos blocos da Fase 2 por participante (Ensino das relações condicionais, revisão da linha de base, testes de simetria, transitividade e de equivalência com estímulos dos conjuntos "A", “B” e “C”).

\begin{tabular}{|c|c|c|c|c|c|c|c|c|}
\hline Bloco & $\begin{array}{l}\text { Ensino } \\
(\mathrm{AB})\end{array}$ & $\begin{array}{l}\text { Simetria } \\
\text { (BA) }\end{array}$ & $\begin{array}{l}\text { Ensino } \\
\text { (AC) }\end{array}$ & $\begin{array}{l}\text { Simetria } \\
\text { (CA) }\end{array}$ & $\begin{array}{l}\text { Revisão } \\
\text { (AB) }\end{array}$ & $\begin{array}{c}\text { Revisão } \\
\text { (AC) }\end{array}$ & $\begin{array}{l}\text { Transiti- } \\
\text { vidade } \\
(\mathrm{BC})\end{array}$ & $\begin{array}{c}\text { Equiva- } \\
\text { lência } \\
\text { (CB) }\end{array}$ \\
\hline & \multirow{5}{*}{$12 / 12$} & & & DRA & \multirow{5}{*}{$12 / 12$} & \multirow{5}{*}{$\begin{array}{l}11 / 12 \\
12 / 12\end{array}$} & \multirow{5}{*}{$12 / 12$} & \multirow{5}{*}{$\begin{array}{l}10 / 12 \\
12 / 12\end{array}$} \\
\hline 1 & & $11 / 12$ & $11 / 12$ & $10 / 12$ & & & & \\
\hline 2 & & \multirow{3}{*}{$12 / 12$} & $10 / 12$ & \multirow{3}{*}{$08 / 12$} & & & & \\
\hline 3 & & & $11 / 12^{*}$ & & & & & \\
\hline 4 & & & $11 / 12^{*}$ & & & & & \\
\hline \multicolumn{8}{|c|}{ LBA } & \\
\hline 1 & & $11 / 12$ & $12 / 12$ & $12 / 12$ & $11 / 12$ & $11 / 12$ & $10 / 12$ & $11 / 12$ \\
\hline 2 & & $10 / 12$ & & & $11 / 12$ & $10 / 12$ & $08 / 12$ & $10 / 12$ \\
\hline \multicolumn{9}{|c|}{ IJA } \\
\hline 1 & $11 / 12$ & $07 / 12$ & $11 / 12$ & $11 / 12$ & \multirow[t]{4}{*}{$12 / 12$} & \multirow[t]{4}{*}{$12 / 12$} & \multirow[t]{4}{*}{$12 / 12$} & \multirow[t]{4}{*}{$12 / 12$} \\
\hline 2 & $12 / 12$ & $04 / 12$ & $12 / 12$ & $11 / 12$ & & & & \\
\hline 3 & & $08 / 12^{*}$ & & & & & & \\
\hline 4 & & $08 / 12^{*}$ & & & & & & \\
\hline
\end{tabular}

* Indica que esse desempenho foi apresentado após o participante ter sido reexposto a blocos separados de relações condicionais do ensino correspondente, conforme programado. 
próxima fase do procedimento. No teste de simetria "CA", atingiu no máximo 10/12 de respostas consistentes com as relações ensinadas (primeiro bloco). Depois das revisões de linha de base "AB" e "AC", foi realizado o teste de equivalência "BC", no qual o participante DRA respondeu com $100 \%$ de respostas consistentes com a linha de base na primeira exposição do teste; seguiu-se o teste "CB", em que o participante mostrou $100 \%$ de consistência na segunda exposição (ver Tabela 1).

O participante LBA atingiu o critério de acerto na linha de base em onze tentativas no total de doze previstas para o teste de simetria "BA" no primeiro bloco. LBA atingiu o critério de acerto na linha de base "AC" e respondeu corretamente no teste de simetria "CA". Mesmo com alguns erros (até dois em cada bloco), o participante respondeu aos testes " $\mathrm{BC}$ " e "CB", acertando dez e onze tentativas respectivamente.
Após atingir o critério de linha de base, IJA respondeu oito tentativas corretamente no bloco de simetria "BA" e onze no bloco "CA". Em um dos blocos do teste de simetria, acertou 4/12 tentativas. Nesse bloco o participante contou a quantidade dos estímulos modelo (estímulos do conjunto "B", que eram numerais cardinais). Posteriormente, solicitou-se que o participante não contasse a quantidade de elementos dos estímulos modelo e sim que respondesse à instrução: "Este (pesquisador referia-se ao estímulo modelo), vai com qual?”, quando do aparecimento dos estímulos de comparação. Essa instrução foi mantida nos demais testes. O participante IJA apresentou responder emergente aos testes de transitividade e de equivalência ( $\mathrm{BC}$ e $\mathrm{CB}$ ). Os resultados da linha de base e dos testes de simetria, transitividade e equivalência podem ser visualizados na Tabela 1.

Tabela 2

Número de acertos sobre o número de tentativas nos blocos de ensino e de sonda durante a aquisição do desempenho de ordenação dos estímulos do conjunto "A".

\begin{tabular}{ccccc}
\hline \multirow{2}{*}{ Tipo de bloco } & Tipo de Tentativa & \multicolumn{3}{c}{ Participante } \\
\cline { 3 - 4 } & & DRA & LBA & IJA \\
\hline Encadeamento & A1 $\rightarrow$ A2 & $3 / 3$ & $3 / 4$ & $3 / 4$ \\
Sonda & $\mathrm{A} 1 \rightarrow \mathrm{A} 2$ & $1 / 1$ & $1 / 1$ & $1 / 1$ \\
Encadeamento & $\mathrm{A} 1 \rightarrow \mathrm{A} 2 \rightarrow \mathrm{A} 3$ & $5 / 6$ & $3 / 3$ & $3 / 3$ \\
Sonda & $\mathrm{A} 1 \rightarrow \mathrm{A} 2 \rightarrow \mathrm{A} 3$ & $1 / 1$ & $1 / 2$ & $6 / 9$ \\
& & & & $0 / 2$ \\
Encadeamento & $\mathrm{A} 1 \rightarrow \mathrm{A} 2 \rightarrow \mathrm{A} 3 / \mathrm{A} 4{ }^{*}$ & $4 / 7$ & $7 / 10$ & $5 / 8$ \\
Sonda & $\mathrm{A} 1 \rightarrow \mathrm{A} 2 \rightarrow \mathrm{A} 3 / \mathrm{A} 4{ }^{*}$ & $1 / 2$ & $4 / 5$ & $1 / 1$ \\
\hline
\end{tabular}

* O estímulo A4 era considerado um estímulo “distrator”, para que último estímulo (A3) não fosse respondido por exclusão. 
Ensino e Teste de Produção de Seqüências

Todos os participantes alcançaram o critério de acerto no ensino por encadeamento com estímulos do conjunto "A". No bloco de tentativas $\mathrm{A} 1 \rightarrow \mathrm{A} 2 \rightarrow \mathrm{A} 3 / \mathrm{A} 4, \mathrm{DRA}$ atingiu o critério na sétima tentativa. A respectiva sonda foi apresentada e esse participante acertou a segunda tentativa. LBA não atingiu o critério de acerto em dez tentativas e o bloco encerrou-se. Na segunda exposição, LBA atingiu o critério na quinta tentativa, já que havia errado a segunda tentativa. IJA errou a tentativa 1, 3 e 5. Esse participante atingiu o critério na oitava tentativa e acertou a respectiva sonda na primeira tentativa. O número de tentativas necessárias para se atingir o critério de acerto pode ser visto na Tabela 2.
Os testes de produção de seqüência, aplicados com estímulos do conjunto "B", foram conduzidos após a revisão de linha de base das relações condicionais $\mathrm{AB} / \mathrm{AC}$ e da sonda $A 1 \rightarrow A 2 \rightarrow A 3 / A 4$. Cada bloco de teste tinha duas tentativas. O participante DRA acertou logo na primeira tentativa. LBA acertou a segunda tentativa da quarta exposição a esse bloco de teste. IJA acertou a segunda tentativa na terceira apresentação do bloco. Todos acertaram a primeira tentativa do teste de produção de seqüência com estímulos do conjunto "C".

Os desempenhos nesses dois testes podem ser visualizados na Tabela 3 .

Após os testes de produção de seqüências "B" e "C", testes de conectividade foram aplicados.

Tabela 3

Número de acertos sobre o número de tentativas nos blocos de testes apresentados após a revisão de linha de base de emparelhamento e encadeamento.

Tipo de bloco Tipo de Tentativa Participante

\begin{tabular}{cccll}
\cline { 3 - 4 } & & DRA & LBA & IJA \\
\hline $\begin{array}{c}\text { Produção de seqüência } \\
\text { Conjunto "B" }\end{array}$ & $\mathrm{B} 1 \rightarrow \mathrm{B} 2 \rightarrow \mathrm{B} 3 / \mathrm{B} 4$ & $1 / 1$ & $0 / 2$ & $0 / 2$ \\
& & & $0 / 2^{*}$ & $0 / 2^{*}$ \\
& & $0 / 2^{*}$ & $1 / 2^{*}$ \\
& & $1 / 2^{*}$ & \\
$\begin{array}{c}\text { Produção de seqüência } \\
\text { Conjunto "C" }\end{array}$ & $\mathrm{C} 1 \rightarrow \mathrm{C} 2 \rightarrow \mathrm{C} 3 / \mathrm{C} 4$ & $1 / 1$ & $1 / 1$ & $1 / 1$ \\
Conectividade "ABC" & $\mathrm{A} 1 \rightarrow \mathrm{B} 2 \rightarrow \mathrm{C} 3 / \mathrm{C} 4$ & $1 / 2$ & $1 / 1$ & $0 / 2$ \\
& & & & $0 / 2^{* *}$ \\
Conectividade"BCA" & $\mathrm{B} 1 \rightarrow \mathrm{C} 2 \rightarrow \mathrm{A} 3 / \mathrm{A} 4$ & $1 / 1$ & $1 / 1$ & $0 / 2$ \\
& & & & $0 / 2^{* *}$ \\
Conectividade "CAB" & $\mathrm{C} 1 \rightarrow \mathrm{A} 2 \rightarrow \mathrm{B} 3 / \mathrm{B} 4$ & $1 / 1$ & $1 / 1$ & $0 / 2$ \\
& & & & $0 / 2^{* *}$ \\
Conectividade "BAC" & $\mathrm{B} 1 \rightarrow \mathrm{A} 2 \rightarrow \mathrm{C} 3 / \mathrm{C} 4$ & $1 / 1$ & $1 / 1$ & $1 / 2$ \\
Conectividade "ACB" & $\mathrm{A} 1 \rightarrow \mathrm{C} 2 \rightarrow \mathrm{B} 3 / \mathrm{B} 4$ & $1 / 2$ & $1 / 2$ & $1 / 2$ \\
Conectividade "CBA" & $\mathrm{C} 1 \rightarrow \mathrm{B} 2 \rightarrow \mathrm{A} 3 / \mathrm{A} 4$ & $0 / 2$ & $1 / 1$ & $0 / 2$ \\
& & & & $0 / 2^{* *}$ \\
\hline
\end{tabular}

* Desempenho apresentado após revisões de linha de base "AB" e da seqüência "A".

** Desempenho apresentado após revisōes de linha de base "AB" e "AC" e da seqüência "A". 
O participante LBA acertou todos os testes de conectividade. LBA respondeu como o esperado logo na primeira tentativa da maioria dos testes, exceto em "ACB".

$\mathrm{O}$ participante DRA acertou a primeira tentativa dos testes "BCA", "CAB" e "BAC", a segunda tentativa dos testes "ABC" e "ACB", mas não acertou o teste "CBA".

O participante IJA acertou os testes "BAC" e "ACB" na segunda tentativa. Mesmo após a revisão de linha de base dos blocos de discriminação condicional e de seqüência, IJA não acertou nenhum dos demais testes.

O número de acertos nos testes de conectividade pode ser visualizado na Tabela 3. Todos os participantes acertaram pelo menos três seqüências em comum na fase de generalização. LBA acertou todas as 9 tentativas, DRA acertou 6 e IJA acertou 5, prontamente ou com emergência gradual (ver Tabela 4).

Tabela 4

Número de acertos sobre o número de tentativas nos testes de generalização com os estímulos dos conjuntos “E”, "F” e "G".

\begin{tabular}{|c|c|c|c|c|}
\hline \multirow[t]{2}{*}{ Bloco de Teste } & \multirow[t]{2}{*}{ Tipo de tentativa } & \multicolumn{3}{|c|}{ Participante } \\
\hline & & DRA & LBA & IJA \\
\hline $\begin{array}{l}\text { Produção de seqüência } \\
\text { Conjunto "E" }\end{array}$ & $\mathrm{E} 1 \rightarrow \mathrm{E} 2 \rightarrow \mathrm{E} 3 / \mathrm{E} 4$ & $1 / 1$ & $1 / 2$ & $\begin{array}{l}0 / 2 \\
1 / 2^{*}\end{array}$ \\
\hline $\begin{array}{c}\text { Produção de seqüência } \\
\text { Conjunto "F" }\end{array}$ & $\mathrm{F} 1 \rightarrow \mathrm{F} 2 \rightarrow \mathrm{F} 3 / \mathrm{F} 4$ & $\begin{array}{l}0 / 2 \\
1 / 1^{*}\end{array}$ & $1 / 1$ & $\begin{array}{l}0 / 2 \\
0 / 2^{*} \\
0 / 2^{*}\end{array}$ \\
\hline $\begin{array}{l}\text { Produção de seqüência } \\
\text { Conjunto "G" }\end{array}$ & $\mathrm{G} 1 \rightarrow \mathrm{G} 2 \rightarrow \mathrm{G} 3 / \mathrm{G} 4$ & $1 / 2$ & $1 / 1$ & $\begin{array}{l}0 / 2 \\
0 / 2^{*} \\
0 / 2^{*}\end{array}$ \\
\hline Conectividade "EFG” & $\mathrm{E} 1 \rightarrow \mathrm{F} 2 \rightarrow \mathrm{G} 3 / \mathrm{G} 4$ & $1 / 2$ & $1 / 1$ & $1 / 2$ \\
\hline Conectividade "FGE” & $\mathrm{F} 1 \rightarrow \mathrm{G} 2 \rightarrow \mathrm{E} 3 / \mathrm{E} 4$ & $\begin{array}{l}0 / 2 \\
1 / 1^{*}\end{array}$ & $1 / 1$ & $\begin{array}{l}0 / 2 \\
0 / 2^{*}\end{array}$ \\
\hline Conectividade "GEF” & $\mathrm{G} 1 \rightarrow \mathrm{E} 2 \rightarrow \mathrm{F} 3 / \mathrm{F} 4$ & $\begin{array}{l}0 / 2 \\
0 / 2^{*}\end{array}$ & $\begin{array}{l}0 / 2 \\
1 / 1^{*}\end{array}$ & $\begin{array}{l}0 / 2 \\
1 / 1^{*}\end{array}$ \\
\hline Conectividade "EGF" & $\mathrm{E} 1 \rightarrow \mathrm{G} 2 \rightarrow \mathrm{F} 3 / \mathrm{F} 4$ & $1 / 1$ & $\begin{array}{l}0 / 2 \\
1 / 1^{*}\end{array}$ & $\begin{array}{l}0 / 2 \\
1 / 1^{*}\end{array}$ \\
\hline Conectividade "FEG" & $\mathrm{F} 1 \rightarrow \mathrm{E} 2 \rightarrow \mathrm{G} 3 / \mathrm{G} 4$ & $0 / 2$ & $1 / 1$ & $\begin{array}{l}0 / 2 \\
0 / 2^{*}\end{array}$ \\
\hline Conectividade "GFE” & $\mathrm{G} 1 \rightarrow \mathrm{F} 2 \rightarrow \mathrm{E} 3 / \mathrm{E} 4$ & $0 / 2$ & $1 / 2$ & $\begin{array}{l}0 / 2 \\
1 / 1^{*}\end{array}$ \\
\hline
\end{tabular}

* Desempenhos apresentados após revisão da seqüência "A". 


\section{DISCUSSÃO}

O objetivo do presente estudo foi investigar se classes ordinais poderiam emergir após o ensino por emparelhamento arbitrário de acordo com o modelo e produção de seqüências. Os resultados apresentados mostraram que as classes formadas a partir do emparelhamento de acordo com o modelo poderiam ser a base para a emergência de classes ordinais. A substitutabilidade de estímulos que caracteriza o fenômeno da equivalência também foi demonstrada na produção de novas sequiências, de acordo com a proposta de Green et al. (1993) de análise de desempenhos emergentes derivados de contingências que estabelecem a produção de sequiências.

Os testes avaliaram se os participantes respondiam a novas seqüências com base na ordinalidade. Após o ensino de relações condicionais $A B / A C$ e da seqüência " $A$ " $(\mathrm{A} 1 \rightarrow \mathrm{A} 2 \rightarrow \mathrm{A} 3 / \mathrm{A} 4)$ por encadeamento de respostas, verificou-se que os participantes realizaram as seqüências " $\mathrm{B}$ " $(\mathrm{B} 1 \rightarrow \mathrm{B} 2 \rightarrow \mathrm{B} 3$ / B4) e "C" ( $\mathrm{C} 1 \rightarrow \mathrm{C} 2 \rightarrow \mathrm{C} 3 / \mathrm{C} 4)$ prontamente ou com emergência gradual (cf. Green \& Saunders, 1998). Os resultados aqui apresentados estendem os estudos anteriores (e. g. Maydack et al., 1995) sobre produção de seqüência e formação de classes ordinais com estímulos numéricos.

A transferência de funçōes discriminativas condicionais para funçôes ordinais e vice-versa tem sido demonstrada por alguns estudos (Green et al., 1991; Lazar, 1977; Lima \& Assis, 2003; Mackay et al., 1997; Maydack et al., 1995; Sigurdardottir et al., 1990). Dentre eles, poucos exploraram a segunda tática descrita por Green et al. (1993). Por exemplo, a principal contribuição no estudo de Lima e Assis (2003) foi demonstrar o controle estabelecido por pareamento consistente sem conseqüências diferenciais imediatas sobre a produção de classes ordinais. Lazar (1977) utilizou seqüências com dois estímulos, o que poderia ter gerado um controle por exclusão (cf. Sigurdardottir et al., 1990). Em Green et al. (1991), adultos responderam aos testes de formação de classes de equivalência por meio de emparelhamento arbitrário em dois estudos. A emergência de classes ordinais após classes de equivalência foi demonstrada prontamente pelo desempenho de dois adultos após o ensino de uma sequiência com instruções mínimas (Estudo 2). Nesse estudo, os autores exploraram o efeito de instruções sobre a transferência de funções ordinais via classes de equivalência estabelecidas. Entretanto, as propriedades ordinais não foram descritas (cf. Green et al., 1993). O uso de testes de conectividade entre seqüências não ensinadas ("B" e "C") e de generalização foi uma contribuição do presente estudo em comparação com os estudos apresentados por Maydack et al. (1995) e Mackay et al. (1997).

O principal ponto em comum com Maydack et al. (1995) foi o procedimento de linha de base de emparelhamento de acordo com o modelo com estímulos numéricos e produção de sequiências. Um dos participantes respondeu prontamente a novas seqüências com formas abstratas (que não faziam parte da história pré-experimental) a partir da linha de base com emparelhamento arbitrário. Esse dado de Maydak et al. (1995) foi similar aos dados com os três participantes do presente estudo, que produziram novas sequiências com estímulos do conjunto "C" (nomes escritos). A ausência de classes ordinais do segundo participante de Maydak et al. (1995) depois do ensino de 
seqüência de quantidades é evidência de que as classes ordinais não se formaram naquele ponto, assim como ocorreu com os participantes LBA e IJA nos primeiros testes da seqüência "B". A adição de estímulos em classes já existentes torna os dados da Fase 1 de Maydak et al. (1995) discutíveis, pois não há controle da história pré-experimental para identificação de todos os pré-requisitos para a formação de classes ordinais desse estudo.

O tipo de procedimento de ensino também é uma variável importante em estudos sobre formação de classes ordinais. Estudos anteriores demonstraram a efetividade no estabelecimento de seqüências por meio de encadeamento de respostas (e.g. Assis \& Costa, 2004; Galy, Camps, \& Melan, 2003; Stromer \& Mackay, 1993). Há dois aspectos relevantes nesse tipo de procedimento. $\mathrm{O}$ primeiro é que os estímulos são adicionados um a um à seqüência e são apresentados simultaneamente de forma que há continuidade de exposição dos estímulos até a formação completa da sequiência. Esse desempenho é mais semelhante ao desempenho final previsto do que aquele estabelecido pelo procedimento de justaposição. Parece que a justaposição é o procedimento mais indicado porque os membros de uma mesma seqüência ensinada nunca apareceram juntos anteriormente (Stromer \& Mackay, 1993; Holcomb, Stromer, \& Mackay, 1997; Lockerbie, Mahon, \& Mackay, 2004). Como o objetivo do presente estudo era verificar a emergência de classes ordinais após o ensino de discriminaçôes condicionais, o encadeamento era o procedimento mais indicado para se garantir a estabilidade da linha de base com a menor probabilidade de erros (cf. Souza \& Assis, 2005).

O controle por propriedades físicas do estímulo como a numerosidade dos elementos do conjunto "A" pode ter se sobressaído, competindo com o controle pela ordem em que o estímulo deveria ser tocado nas seqüências de teste. Os participantes DRA e LBA apresentaram os desempenhos previstos, com a emergência de classes ordinais. Entretanto, o participante IJA contava os elementos dos estímulos durante o ensino por encadeamento de respostas. Essa resposta foi mantida mesmo em seqüências de teste em que o controle pela posição do estímulo na seqüência deveria se sobressair, em detrimento da numerosidade de elementos dos estímulos do conjunto "B" e "C".

Uma alternativa para isso foi o estudo apresentado por Silva e Galvão (2001), que demonstraram que erros ocorreram com mais freqüência em conjuntos desalinhados (elementos com disposição espacial irregular) do que alinhados (elementos com disposição espacial regular). Os dados do presente estudo confirmam esses resultados, já que os participantes apresentaram mais erros em ordenar estímulos cujos elementos eram desalinhados do que estímulos com elementos alinhados. Além disso, a contagem oral pode ser facilitadora para a generalização do comportamento de ordenar estímulos com base na numerosidade (cf. Monteiro \& Medeiros, 2002).

Dentre as relações componentes do comportamento conceitual numérico descritas por Carmo (2002), algumas foram estabelecidas: diante de uma coleção de objetos, escolher dentre dois ou mais nomes escritos de numerais, aquele que corresponde à quantidade apresentada; diante de uma coleção de objetos escolher dentre dois ou mais numerais, aquele que corresponde à quantidade apresentada (de um a três). Com relação às seqüências numéricas, os desempenhos estabelecidos foram: ordenar quantidades de um a quatro, 
em seqüência crescente. Esses desempenhos foram apresentados por todos os participantes na linha de base do presente estudo.

Os resultados mostraram que após o ensino de relações numéricas, a emergência de classes ordinais tem alta probabilidade de ocorrer, uma vez que as funçôes estabelecidas por um membro da classe no contexto de discriminação condicional, são transferidas para todos os membros da classe ordinal. Porém algumas contingências de reforçamento são fundamentais, como por exemplo: uma linha de base de emparelhamento arbitrário em que o controle discriminativo seja pelo $\mathrm{S}_{+}$; tarefas de emparelhamento de acordo com o modelo e de produção de seqüências relacionadas. Para o primeiro requisito, alguns autores (cf. McIlvane \& Dube, 2003) sugerem o uso de procedimentos específicos para a verificação do controle pelo $S+$ ou $S$-, o que não foi feito neste estudo. Para o segundo requisito, o tipo de instrução pode ser uma variável importante (cf. Green et al., 1991).

Neste estudo, os resultados dos testes de generalização permitem afirmar que não havia outra fonte de controle concorrente, uma vez que esse responder ocorreu prontamente ou gradualmente, com a transferência de funçôes ordinais para os novos estímulos. Estudos mais sistemáticos com essas variáveis poderiam explicitar controles mais precisos do responder ordinal. Sugerese ainda, para estudos futuros, que sejam usadas formas abstratas, devendo-se manter uma única dimensão relevante do estímulo para um controle experimental mais efetivo. Uma outra possibilidade seria aplicar o teste de generalização com elementos do ambiente escolar do participante.

\section{REFERÊNCIAS}

Assis, G. J. A., \& Costa, L. C. A. (2004). Emergência de relações ordinais em crianças. Interação em Psicologia, 8, 199-216.

Carmo, J. S. (2002). Definiçôes operacionais de habilidades matemáticas elementares Em: H. J. Guilhardi (Ed.), Sobre o comportamento e cognição: Contribuições para a construção da teoria do comportamento (pp. 181-191). São Paulo: ESETEC.

Cumming, W. W., \& Berrymann, R. (1965). The complex discriminated operant: Studies of matching-to-sample and related problems. Em: D. I. Mostofsky (Ed.), Stimulus Generalization (pp.284-330). Stanford: Stanford University Press.

Dube, W. (1996). Teaching discrimination skills to persons with mental retardation. Em C. Goyos, M. A. Almeida, \& D. G. de Souza (Orgs.), Temas em Educação Especial (pp. 73-96). São Carlos: EDUFScar.

Dunn, L. M., \& Dunn, I. M. (1981). Peabody Picture Vocabulary Test-Revised. Circle Pines, MN: American Guidance Service.

Galy, E., Camps, J. F., \& Melan, C. (2003). Sequence class formation following learning of short sequences. The Psychological Record, 53, 635-645.

Green, G., \& Saunders, R. R. (1998). Stimulus equivalence. Em K. Lattal, \& M. Perone, (Eds.) Handbook of research methods in human operant behavior (pp. 229-262). New York: Plenum Press. Green, G., Sigurdardottir, Z. G., \& Saunders, R. R. (1991). The role of instructions in transfer of ordinal functions through equivalence classes. Journal of the Experimental Analysis of Behavior, 55, 287-304.

Green, G., Stromer, R., \& Mackay, H. (1993). Relational learning in stimulus sequences. The Psychological Record, 43, 599-616.

Holcomb, W. L., Stromer, R., \& Mackay, H. (1997). Transitivity and emergent sequence performance in young children. Journal of Experimental Child Psychology, 65, 96-124. 
Lazar, R. (1977). Extending sequence-class membership with matching to sample. Journal of the Experimental Analysis of Behavior, 27, 381-392.

Leite, S. A. S. (1984). Instrumento de Avaliação do Repertório Básico para Alfabetização. São Paulo: Edicon.

Lima, M. P. L., \& Assis, G. J. A. (2003). Emergência de classes seqüenciais após treino com pareamento consistente. Psicologia: Teoria e Pesquisa, 19, 75-84.

Lockerbie, A. A. M., Mahon, K. L., \& Mackay, H. (2004). Emergent numeric sequence performances in children with intellectual disabilities. Proceedings of the 30th Annual Convention of the Association Behavior Analysis, Boston, MA.

Mackay, H., Kotlarchyk, B., \& Stromer, R. (1997). Stimulus classes, stimulus sequences, and generative behavior. Em D. Baer, \& E. M. Pinkston, (Eds.) Environment and behavior (pp. 124-137). Boulder, CO: Westview Press.

Maydak, M., Stromer, R., Mackay, H., \& Stoddard, L. (1995). Stimulus classes in matching to sample and sequence production: the emergence of numeric relations. Research in Developmental Disabilities, 16, 179-204.

McIlvane, W. J., \& Dube, W. V. (2003). Stimulus control topography coherence theory: Foundations and extensions. The Behavior Analyst, 26, 195-213

Monteiro, G., \& Medeiros, J. G. (2002). A contagem oral como pré-requisito para a aquisição do conceito de número com crianças pré-escolares. Estudos de Psicologia, 7, 73-90.

O’Donnell, J., \& Saunders, K. J. (2003). Equivalence relations in individuals with language limitations and mental retardation. Journal of the Experimental Analysis of Behavior, 80, 131-157.

Saunders, K. J., \& Spradlin, J. (1990). Conditional discrimination in mentally retarded adults: The development of generalized skills. Journal of the
Experimental Analysis of Behavior, 54, 239-250

Sidman, M. (1986). Functional analysis of emergent verbal classes. Em: T. Thompson \& M. D. Zeiler (Eds.). Analysis and integration of behavioral units (pp. 213-245). Hillsdale, New Jersey: Lawrence Erlbaum Associates Publishers.

Sidman, M. (1994). Equivalence relations and behavior: A research story. Boston: Authors Cooperative Inc. Publishers.

Sidman, M., \& Tailby, W. (1982). Conditional discrimination versus matching to sample: and expansion of the testing paradigm. Journal of the Experimental Analysis of Behavior, 37, 5-22.

Sigurdardottir, Z. G., Green, G., \& Saunders, R. R. (1990). Equivalence classes generated by sequence training. Journal of the Experimental Analysis of Behavior, 53, 47-63.

Silva, L. C. C., \& Galvão, O. F. (2001). Tipo de elemento e desempenho em tarefas de contagem. Em: R.M. E. Figueiredo, L. C. Silva, U. R. Soares, U. R., \& R. S. Barros (Eds.). Ensino da leitura, escrita e conceitos matemáticos (pp. 89-132). Belém: Universidade da Amazônia.

Souza, J. A. N., \& Assis, G. J. A. (2005). Efeito de dois procedimentos de ensino sobre o comportamento de ordenar. Psicologia em Estudo, 10, 527-536.

Stromer, R., \& Mackay, H. (1993). Human sequential behavior: Relations among stimuli, class formation and derived sequences. The Psychological Record, 43, 107-131.

Stevens, J. (1951). Mathematics, measurement, and psychophysics. Em: S. Stevens (Ed.), Handbook of Experimental Psychology (pp. 1-49). New York: John Wiley and Sons, Inc.

Submetido em 12 de janeiro de 2006 Aceito em 22 de novembro de 2006 


\title{
PROGRAMAS DE PÓS-GRADUAÇÃO STRICTO SENSU EM ANÁLISE DO COMPORTAMENTO NO BRASIL BRAZILIAN GRADUATE PROGRAMS IN BEHAVIOR ANALYSIS
}

\author{
PROGRAMA DE PÓS-GRADUAÇÃO EM PSICOLOGIA \\ Centro de Educação e Ciências Humanas \\ Universidade Federal de São Carlos \\ Cursos: MESTRADO E DOUTORADO \\ Área de concentração \\ Comportamento e Cognição \\ Linhas de Pesquisa \\ 1. Análise comportamental da cognição \\ 2. Comportamento social e processos cognitivos \\ COORDENADORA: DEISY DAS GRAÇAS DE SOUZA
}

\section{DOCENTES:}

Almir Del Prette

Antonio Celso de Noronha Goyos

Azair Liane Mattos do Canto de Souza

Camila Domeniconi

Deisy das Graças de Souza

Elizabeth Joan Barham

Júlio César Coelho de Rose

Lúcia Cavalcanti de Albuquerque Williams

Maria Stella Coutinho de Alcântara Gil

Rosemeire Aparecida Scopinho

Susi Lippi Marques Oliveira

Zilda Aparecida Pereira Del Prette

Informações adicionais na página da internet: www.ppgpsi.ufscar.br

E-mail:ppgpsi@power.ufscar.br 